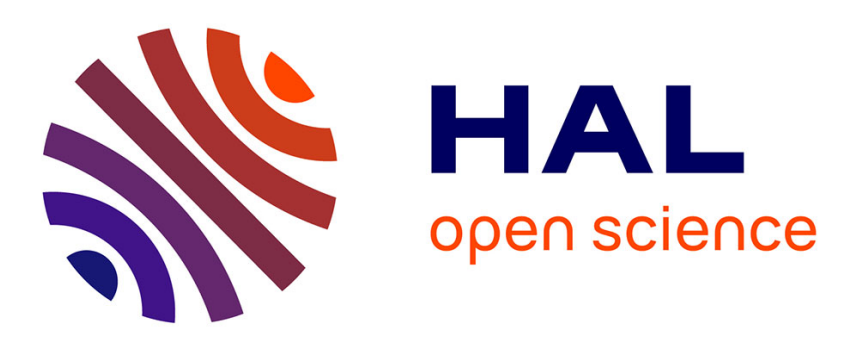

\title{
Arrien avant Arrien: une jeunesse entre Bithynie, Grèce et Rome
}

\author{
Dan Dana, Madalina Dana
}

\section{To cite this version:}

Dan Dana, Madalina Dana. Arrien avant Arrien: une jeunesse entre Bithynie, Grèce et Rome. KTÈMA Civilisations de l'Orient, de la Grèce et de Rome antiques, 2014, 39, pp.19-35. halshs01405116

\section{HAL Id: halshs-01405116 https://shs.hal.science/halshs-01405116}

Submitted on 29 Nov 2016

HAL is a multi-disciplinary open access archive for the deposit and dissemination of scientific research documents, whether they are published or not. The documents may come from teaching and research institutions in France or abroad, or from public or private research centers.
L'archive ouverte pluridisciplinaire HAL, est destinée au dépôt et à la diffusion de documents scientifiques de niveau recherche, publiés ou non, émanant des établissements d'enseignement et de recherche français ou étrangers, des laboratoires publics ou privés. 


\title{
version auteur avant publication
}

\author{
Arrien avant Arrien : \\ une jeunesse entre Bithynie, Grèce et Rome
}

\begin{abstract}
RÉsumÉ-. S'intéresser à la jeunesse et à la formation d'Arrien, avant qu'il ait connu la célébrité, représente, plus qu'une analyse d'un segment de sa vie et de son œuvre, de son attachement à sa patrie Nicomédie, où il assuma son rôle de notable, et en général à sa Bithynie, une occasion de dépasser la dichotomie traditionnelle d'un Arrien placé entre Grèce et Rome. L'image qui se dégage est certes plus complexe et invite à revisiter la documentation littéraire et épigraphique, compte tenu du dynamisme culturel qui caractérise la province de Pont-Bithynie à l'époque impériale. Les fragments de son histoire locale, les Bithyniaques, qui inscrivent sa région dans le passé légendaire comme dans l'histoire globale, à l'aide de nombreuses allusions mythologiques et littéraires, montrent à quel point les référents hellénique et romain sont indissociables de son origine provinciale.
\end{abstract}

AвSTRACT-. Studying Arrian's youth and formation, before knowing the celebrity, represents, more than an analysis of a segment of his life and work, or of his attachment to his homeland Nicomedia, where he assumed his role of notable, and generally to his Bithynia, an opportunity to surpass the traditional dichotomy of an Arrian between Greece and Rome. The corresponding image is certainly more complex and asks to revisit the literary and epigraphic evidence, taking into account the cultural dynamism of the province of PontusBithynia during the imperial period. The fragments of his local history, Bithyniaca, inscribing his region in the legendary past as well as in the global history, using therefore numberless mythological and literary allusions, shows to what extent the Hellenic and Roman identities are inseparable of his provincial origin.

Cet article consacré à la jeunesse d'Arrien ${ }^{1}$ avant qu'il ait connu la célébrité, d'où le titre «Arrien avant Arrien», essaiera, même s'il s'agit d'un segment d'une longue vie (ca. 85/90-après 161 ap. J.-C.), de dépasser la dichotomie désormais traditionnelle, d'un personnage placé entre deux mondes, Grèce et Rome, tel qu'il apparaît chez de nombreux auteurs ${ }^{2}$, alors que la réalité se révèle bien plus complexe ${ }^{3}$. Connu comme «Arrien le philosophe» par ses contemporains, mais qualifié d' «historien» par les Modernes, qui ne cessent d'exploiter et d'analyser son Anabase d'Alexandre,

(1) Les traductions du résume des Bithyniaques d'Arrien dans la Bibliothèque de Photius sont celles de R. Henry, modifiées; sauf mention contraire, les numéros des fragments des Bithyniaques sont ceux de l'édition de Roos, suivis parfois des références à l'édition de F. Jacoby (=J.). Nous remercions vivement Konrad Stauner, qui nous a fait parvenir l'article récent sur les Flavii Vlpii de Nicomédie, ainsi qu'Antony Hostein, pour ses suggestions toujours utiles.

(2) Entre autres, Vidal-Naquet 1984, «Flavius Arrien entre deux mondes»; Bosworth 1993, p. 226-233, «Astride two Worlds»; Swain 1996, p. 242-248, "Arrian - Roman Politics and Greek Culture»; Madsen 2009, p. 119-123, «L. Flavius Arrianus: A Roman Authority and a Nostalgic Greek».

(3) Voir l'analyse nuancée d'Antony Hostein dans ce dossier. 
Arrien fut un auteur prolifique. En effet, la plupart de ses ouvrages furent conservés et transmis par ses lecteurs tardo-antiques et byzantins en raison de sa célébrité. Nous avons choisi d'évoquer sa première patrie, généralement négligée dans les études sur Arrien ${ }^{4}$ en raison notamment de la documentation éparse ou ambiguë: on s'intéressera d'abord à ses années d'études et de formation, puis on traitera de sa carrière à Nicomédie, qui fut sa patrie, et des liens qu'il a pu conserver par la suite avec sa région natale, pour finir par une analyse de son histoire locale, la monographie de la Bithynie. Ses Bithyniaques, l'une des innombrables histoires locales qui n'ont pas eu le rare privilège d'être conservées par la tradition directe, sont connus essentiellement par le résumé du patriarche Photius et par des échos d'autres érudits byzantins, à une époque où la Bithynie se trouvait dans la proximité immédiate du centre du pouvoir, Constantinople. Autant dire qu'ils ne nous livrent pas toute l'information à laquelle Arrien a eu accès pour rendre hommage à la terre de ses ancêtres, mais nous laissent entrevoir la curiosité et parfois la fierté qui l'ont mené dans cette entreprise identitaire.

\section{LA JEUNESSE STUDIEUSE D’ARRIEN DE NICOMÉDIE: UNE REMISE DANS LE CONTEXTE}

À vrai dire, peu de choses sont connues sur la jeunesse d'Arrien ${ }^{5}$, car ses biographies anciennes sont perdues, tel l'ouvrage de Dion Cassius, un autre Bithynien avec une carrière et des occupations similaires, originaire quant à lui de la voisine Nicée, et qui avait écrit au $\mathrm{III}^{\mathrm{e}}$ siècle une Vie du philosophe Arrien ${ }^{7}$. Les témoignages littéraires conservés sont peu bavards et les mentions épigraphiques concernent d'autres épisodes de son parcours.

Arrien, de son nom complet L. Flavius Arrianus, naquit à Nicomédie ${ }^{8}$, dans une famille de notables pratiquement inconnue, qui a sans doute reçu la citoyenneté romaine sous les Flaviens ${ }^{9}$, ce qui explique le gentilice Flavius ${ }^{10}$. Quant au cognomen, les commentateurs ont suggéré, puisqu'il s'agit d'un dérivé en -anus du gentilice Arrius, un possible mariage de l'un de ses prédécesseurs avec un Arrius ou une Arria. Mais, ce n'est pourtant qu'une hypothèse: si le nom Appıavóc n'est pas très fréquent dans l'Orient grec, on dispose désormais d'un bon point de départ avec le tome V.A du $L G P N$. On remarque aisément une relative popularité de ce nom en Bithynie et en Mysie voisine, mais aucune occurrence dans le Pont Sud ou sur la côte égéenne, alors que de nouvelles découvertes épigraphiques ${ }^{11}$ renforcent l'ancrage local de ce nom prisé dans les contrées bithyniennes, où il présente une douzaine d'occurrences.

(4) Seul Madsen 2009, p. 121, évoque une identité bithynienne distincte, en plus des deux mondes.

(5) Sur la vie et l'œuvre d'Arrien (PIR ${ }^{2}$ F 219), voir F. JACOBY, FGrHist, II.B. Komm., 1930, p. 551-553; Bowersock 1969, p. 113 (Arrien et Appien, comparés avec leur milieu sophistique); BowIE 1970, p. 24-28; BosworTH 1972 et 1980 (I, p. 1-7); Stadter 1980; Vidal-Naquet 1984; Bosworth 1988, p. 16-37 (sa production historique); Syme 1988; ToNNET 1988, I, p. 7-101; Follet 1989; Bosworth 1993, p. 226-233 (ses ouvrages mineurs, relativement négligés); Devine 1993, p. 312-315; SwaIn 1996, p. 242-248; Fernoux 2004, p. 499-501; MADSEN 2006, p. 70-71 et 2009, p. 68-70 et 196-123.

(6) Sur l'éternelle rivalité Nicée-Nicomédie, voir Robert 1977 (=OMS, VI, p. 211-249).

(7) Dion Cassius, Vie du philosophe Arrien (FGrHistCont 1075=Arrien, FGrHist 156 T 8=T 1 Roos), cf. Souda, s.v.

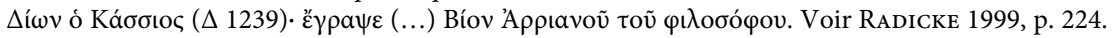

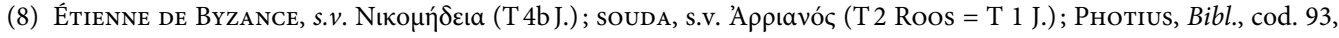
71a (T3 Roos=T4a J.); Jérôme (=Eusèbe de CÉsarée), I, p. 203 Helm (T22 Roos); Georges le Syncelle, p. 662, 18 Bonn. (T22 Roos = T3 J.).

(9) Ou bien avant, car il existe plusieurs hypothèses, en rapport aussi avec le praenomen, sans doute Lucius.

(10) Alors que Stadter 1967 pensait à un second nom («Flavius Arrianus, the New Xenophon»), Ameling 1984a («L. Flavius Arrianus Neos Xenophon») prouve qu'il s'agit en réalité d’un surnom honorifique.

(11) Voir LGPN V.A 68: en tout, 7 occurrences, dont une dans le voisinage, à Hadrianeia en Mysie. Nous pouvons

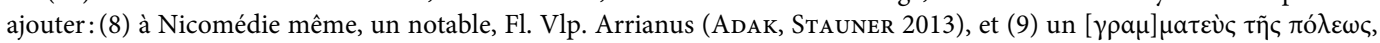


Tournons-nous à présent vers la jeunesse studieuse d'Arrien. De ses propres mots, il était né et avait été éduqué ( $\pi \alpha \iota \delta \varepsilon v \theta \tilde{\eta} v a l)$ à Nicomédie, selon le résume du patriarche Photius: «il précise, en effet, dans [les Bithyniaques] que sa famille est de Nicomédie et que c'est dans cette ville qu'il est né, qu'il a été élevé et instruit ${ }^{12}$. Arrien a dû fréquenter le gymnase local, dans une cité qui s'impose comme une grande ville marchande, à vocation maritime, avec de nombreux ressortissants attestés dans le monde méditerranéen, spécialisés dans la navigation, mais aussi dans le travail de la pierre et du bois ${ }^{13}$. Qui plus est, forte de son passé de capitale de l'ancien royaume de Bithynie, Nicomédie a également fourni un nombre non négligeable d'intellectuels ou de professionnels du savoir ${ }^{14}$, dont nous avons dressé une liste actualisée. On connaît ainsi, à Nicomédie même, en dépit du caractère épars de la documentation épigraphique:

- le géomètre Markianos fils de Hippias ${ }^{15}$;

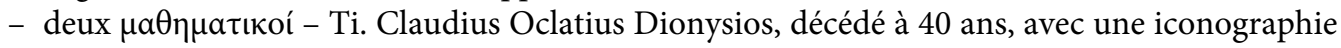
funéraire intéressante: entre le défunt, assis sur un siège (diphros, sorte de chaise curule) et représenté dans l'attitude méditative du philosophe, et son épouse, se dresse une table sur laquelle est posé un rouleau ${ }^{16}$; l'épitaphe inédite de Numitorius (?) Acutianus ${ }^{17}$;

- deux médecins: [-] Rubius Iulianus, décédé à 60 ans $^{18}$; Flavius Attikos ${ }^{19}$;

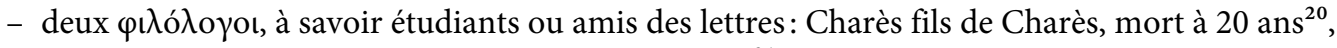
et $\mathrm{P}$. Aelius Antonianus Theodosios, mort à $22 \mathrm{ans}^{21}$;

- un philosophe et bouleute, Aur. Dèmètrios, qui consacra au $\mathrm{III}^{\mathrm{e}}$ siècle une statuette inscrite

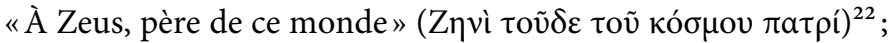

- un autre philosophe, Maximianos (?), connu par une stèle funéraire aujourd'hui perdue qui a été décrite par un voyageur anglais du XvII ${ }^{\mathrm{e}}$ siècle, John Covel; elle portait un relief suggestif, puisque le défunt était représenté debout, dans la posture d'un lettré, un rouleau à la main ${ }^{23}$.

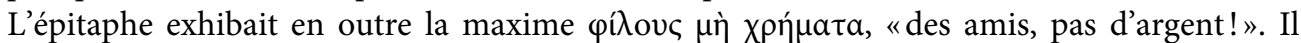
convient d'ajouter qu'on retrouve cette maxime, plus développée, dans deux gnomologes tardifs attribués à Épictète ${ }^{24}$, ainsi qu'un autre parallèle dans le Manuel d’Epictète: $\tau$ 'í $\delta \grave{~}$ kaì

Longinianus Arrianus (inscription inédite, cf. Adak, STAUner 2013, p. 145 n. 5); (10-11) deux occurrences de la variante simplifiée Ápıаvó ৎ dans le territoire de Nicomédie (LGPN V.A 59), et (12) une autre dans le territoire de Nicée (ÖzTÜRK, KILIÇ Aslan 2012, p. 108, n 14). Signalons à ce propos, dans la notice Appıðvó ( $L G P N$ V.A 68), une erreur des rédacteurs, puisque le $\mathrm{n}^{\circ} 2$ (décret honorifique, SEG I 446a =TAM IV.1 41a) et le $\mathrm{n}^{\circ} 3$ (données prosopographiques) se rapportent à notre Arrien le philosophe. Appıavó s peut aussi bien être porté par des pérégrins, comme idionyme, cf. l'épitaphe du territoire de Nicomédie d'Arrianos fils de $\Delta$ ot $\delta a \lambda \sigma \eta \varsigma$ (nom indigène) (TAM IV.1 182).

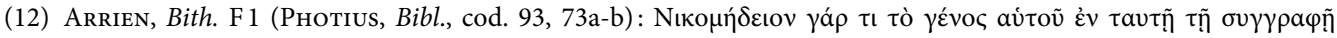

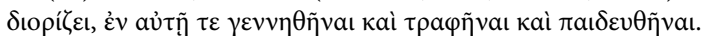

(13) Voir Robert 1960, p. 35-36 et 1987, p. 408-428 («Épitaphes de Nicomédie»); Bounegru 2006.

(14) Pour les intellectuels bithyniens d'époque impériale, voir Fernoux 2004, p. 490-495; BekKer-Nielsen 2008 et MareK 2013 (sur Dion Chrysostome).

(15) TAM IV.1 173.

(16) Dörner 1952, p. 96, nº 101 (photo Pl. 36) = TAM IV.1 200; Fernoux 2004, p. 493.

(17) Cf. TAM, IV.1, p. 51.

(18) TAM IV.1 135 = SAMAMA 2003, nº308.

(19) TAM IV.1 220 = SAMAMA 2003, n³07.

(20) TAM IV.1 155; signalons le nom indigène de sa mère, TIT $\Theta$ A

(21) TAM IV.1 232.

(22) SEG XXXII 1255 (à Gügüşler, dans le territoire de Nicomédie); voir PUECH 1994, p. 628.

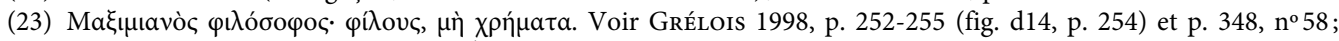
SEG XLIX 1792 et LII 1234 (sur le nom); $A E$, 1998, 1286; PUeCH 2005, p. 303. Les lectures du nom du défunt divergent:

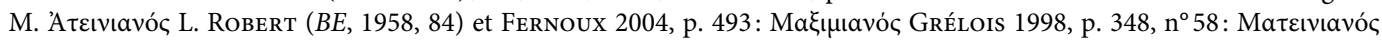

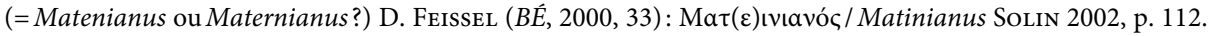

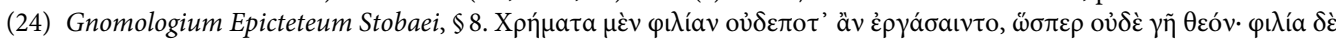

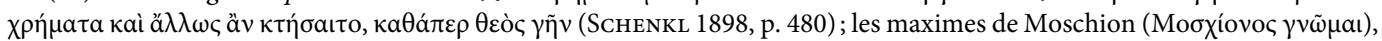




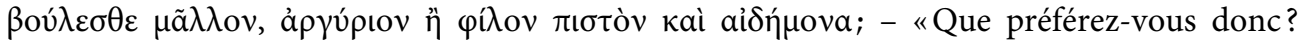
De l'argent ou un ami fidèle et réservé ? ${ }^{25}$. Maximianos était donc un philosophe stoïcien d'obédience épictétienne, à l'instar de son compatriote Arrien, comme nous aurons l'occasion de le constater.

Deux épigrammes funéraires de Nicomédie, récemment publiées, nous font connaître deux parcours différents. D’abord, celui de Basilikos, qui avait reçu les sages enseignements de la terre

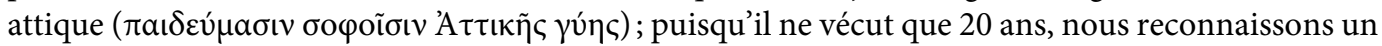
autre $\varphi \iota \lambda o ́ \lambda o \gamma o \varsigma$ parti parfaire son éducation ailleurs, dans le cas présent à Athènes. Le second est celui de Kyriôn, bouleute de la " métropole Nicomédie», décédé avant 70 ans, qui est défini par ses

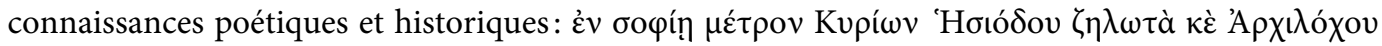

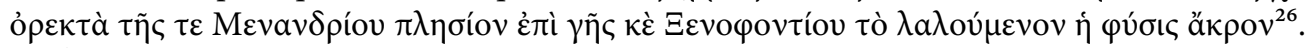

À l'étranger, sans préjudice de nombreux athlètes et musiciens, plusieurs lettrés et spécialistes du savoir originaires de Nicomédie sont connus à l'époque impériale, la patrie d'Arrien participant ainsi au dynamisme culturel bithynien, qui se manifeste avec vigueur dès la basse époque hellénistique:

- au moins quatre médecins: Euandros à Lambèse, en Afrique du Nord ${ }^{27}$; Q. Aelius Archelaos, décédé à 22 ans à Rome ${ }^{28}$; M. Octavius Firmi f. Pal(atina tribu) Aper, Nicom(edia), medicus (militaire?), connu par une épitaphe de Pliska, en Mésie Inférieure, où apparaît également son frère M. Octavius [Pal(atina tribu)] D(o]mitius, Nicom(edia), vet(eranus) [l]eg(ionis) $V$ Mac(edonicae ${ }^{29}$; Ménodote, médecin $\varepsilon \dot{\mu} \pi \varepsilon ı$ ıкó , qui était aussi philosophe sceptique (ca. 125 ap. J.-C.) ${ }^{30}$;

- un architecte anonyme, devenu citoyen de Tomi, métropole du Pont Gauche, en Mésie Inférieure. A l'instar de nombreux autres Bithyniens ${ }^{31}$, il y avait professé, ainsi qu'à Olbia du Pont où il est attesté à l'extrême fin du $\mathrm{II}^{\mathrm{e}}$ siècle $^{32}$;

- un juriste (iuris studiosus), T. Oclatius Athenagora, mort à Rome ${ }^{33}$;

- deux $\gamma \rho \alpha \mu \mu \alpha \tau$ เкó́: Myrsinos à Philippopolis, en Thrace ${ }^{34}$; Asklèpiodôros, visiteur à Thèbes d'Égypte $^{35}$;

- l'orateur P. Aelius Kleisthenès, mort le plus probablement à Smyrne, à 28 ans ${ }^{36}$;

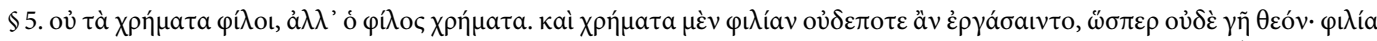

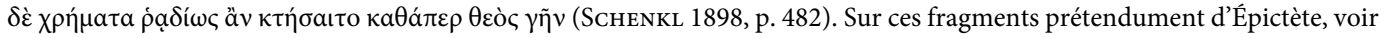
Fuentes GoNZÁLEZ 2000, p. 119.

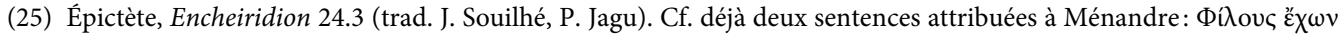

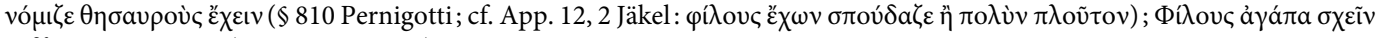

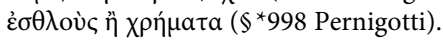

(26) AкyÜrek ŞAhin 2011; avec les observations critiques de P. Hamon, BÉ, 2012, 415 et de Jones 2014, p. 29-33 (nº XI).

(27) Robert 1939, p. 166-167 (= OMS, II, p. 1319-1320); Laronde 2002-2003; Samama 2003, nº 458.

(28) IG XIV $2019=$ IGVR II $299=$ SAMAMA $2003, \mathrm{n}^{\circ} 464$

(29) ConRad 2004, p. 211-212, n 316.

(30) Boudon-Millot 2005. Des philosophes stoïciens sont attestés par l'épigraphie à Pruse de l'Olympe (I. Prusa ad Olympum 17 et 18) et ailleurs en Bithynie, voir Th. Consten, I. Prusa ad Olympum, p. 33-34.

(31) Voir l'étude spéciale d'Avram $2013 \mathrm{~b}$.

(32) IGR I $84=$ IOSPE I² 174; DANA 2011 (p. 202) et 2012, p. 256-257; Avram 2013a, p. 301, nº 3146.

(33) CIL VI $33868=$ ILS 7742.

(34) IGB III.1 1021.

(35) BAillet, no 1739.

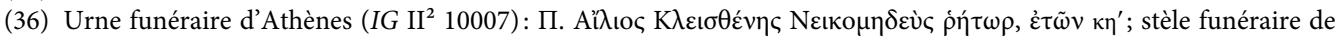

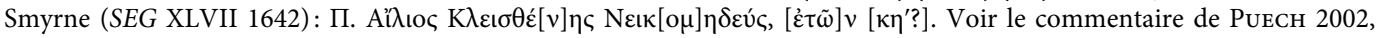
p. $176-177, n^{\circ} 66-67$. 
- deux sophistes:P. Aelius Samios Isokratès, également citoyen d'Éphèse, décédé à Ostie ${ }^{37}$, et le chevalier Quirinus, élève d'Hadrien de Tyr à Éphèse, advocatus fisci d'une grande modération dans la province d'Asie, enterré dans sa patrie ${ }^{38}$;

- en ce qui concerne les philosophes ${ }^{39}$, outre le médecin empirique Ménodote, deux «deipnosophistes» mentionnés par Athénée, Dèmokritos et Pontianos ${ }^{40}$, pourraient être des personnes ayant réellement existé;

- enfin, un $\varphi \imath \lambda o ́ \lambda o \gamma o \varsigma$, Prousès, fils de Ménékratès, mort à 24 ans à Mégare, sur lequel on reviendra. Il s'agit d'un terme technique employé pour désigner une catégorie studieuse ${ }^{41}$.

Cette présence notable de lettrés nicomédéens à l'étranger, exerçant déjà une profession ou tout simplement en train de parfaire leur éducation, nous aidera à mieux situer le parcours d'Arrien. Vers $108^{42}$, entre les guerres daciques et les guerres parthiques, il assista aux leçons d'Épictète (ca.50-125/130 $)^{43}$, à Nicopolis d'Épire, et commença à fréquenter Athènes, puisqu'il fut initié, paraît-il, à Éleusis. Cette expérience le mettra en contact avec des cercles du pouvoir dans la province d'Achaïe vers 112 ou $113^{44}$, facilitant ainsi sa carrière militaire sous Trajan et, un peu plus tard, son cursus sénatorial.

On peut attribuer à Arrien une contribution essentielle à l'histoire de la philosophie antique. En effet, c'est essentiellement grâce à lui que l'on connaît la doctrine d'Épictète, dont il fut auditeur et disciple. Arrien se chargea de publier ses notes de cours en deux recueils:

- les Entretiens, $\Delta$ ıа au lieu de huit), qui sont précédées par une lettre-préface à un certain L. Gellius; le dédicataire est sans doute issu de la grande famille des Gellii de Corinthe, peut-être L. Gellius Iustus, fils de L. Gellius Ménandros ${ }^{45}$. Ces Entretiens sont présentés comme la transcription fidèle des cours de son maitre. L'on apprend même qu'Arrien fut amené à en donner une édition "officielle», peut-être entre 125 et 130, après avoir constaté la circulation de plusieurs copies

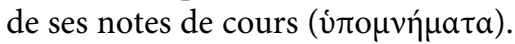

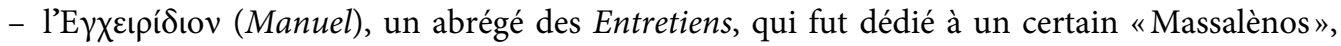
d'après la lettre-préface, perdue, qui est mentionnée dans le commentaire de Simplicius ${ }^{46}$.

(37) SEG XII $472+$ XXX 1178; PUECH 2002, p. 447, nº 236.

(38) Philostrate, VS2.29; Follet 2012.

(39) À l'époque hellénistique déjà, on connaît plusieurs membres de l'Académie originaires de Nicomédie, tous disciples du scholarque Carnéade: Diogène (Dorandi 1994), Euclide (Dorandi 2000a), Hérodote (Dorandi 2000b) et Sarapiôn.

(40) Goulet 1994; Fuentes GonzÁlez 2012.

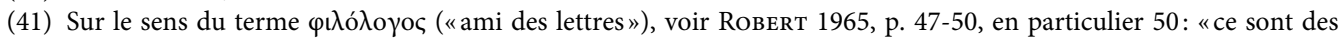
jeunes gens ou petits jeunes gens, des étudiants, et non des professionnels enseignant la philologie; d'où leur présence dans les grandes villes d'enseignement de Smyrne et d'Éphèse. Le mot est un éloge faisant allusion à leurs études, non la mention d'un professeur».

(42) Fuentes González 2000 (avec les diverses hypothèses); en faveur de cette date est invoquée la mention, chez Pline le Jeune (Ep. 8.24.2-4), d'un épicurien du nom de Maximus comme corrector des cités libres, qui aurait fréquenté l'école d'Épictète (voir Syme 1985, p. 329-331).

(43) Fuentes González 2000.

(44) Ainsi, à Delphes, Arrien fait partie du consilium d'un haut personnage, le gouverneur d'Achaïe C. Avidius Nigrinus (PIR A 1407); ce dernier, consul suffect en 110, cultivé et philhellène, était ami d'Hadrien (voir FD III.4 290, 291 et 294; Meritt 1963, p. 24, n² 25, 1. 10). On ignore s'il avait participé aux guerres parthiques de Trajan; GrassL 1982, p. 245-252, suppose une inspection dans les années 120 du limes rhénan et danubien avec Hadrien; voir aussi WiRTH 1974.

(45) Roos 1967, II, p. 196. Vers la même époque, Arrien est honoré à Corinthe comme bienfaiteur par L. Gellius Menander et L. Gellius Iustus f(ilius), en qualité de «philosophe et légat propréteur de l'empereur Hadrien en Cappadoce». Voir CIL III 7269; Corinth VIII.2 93, VIII.3 124 et 125; SEG XXXI 285; cf. Oliver 1970, p. 335-338; Follet 2000.

(46) Simplicius, Comm. in Epict. Ench., praef. init. (=T 4 Roos=F 53 J.); il pourrait s'agir de C. Vlp. Pacatus Prastina Messalinus (PIR ${ }^{2}$ M 512), consul en 147, voir GOULET 2005. 
On ignore les circonstances précises du choix de son maître - outre la célébrité d'Épictète - et de Nicopolis d'Épire ${ }^{47}$, sinon le désir de parfaire son éducation ailleurs que dans sa patrie, selon une pratique qui se généralise à l'époque et qui est bien attestée pour ses compatriotes. On ignore également tout ce qui pouvait aider de manière plus concrètele jeune Arrien: sa famille et son indispensable support financier ${ }^{48}$, des amis, d'autres Bithyniens - un " réseau bithynien" qui s'active sans doute sous Hadrien, comme le remarque Antony Hostein. Un autre Bithynien tout aussi célèbre, Dion de Pruse, dit Chrysostome ${ }^{49}$, qui connaît son acmé au début du règne de Trajan, s'était formé auprès du stoïcien Musonius Rufus, maitre d'Epictète, exilé par Néron en Asie: Dion devait donc connaître Épictète, son condisciple, qui dépassa son maître. Cette expérience avait permis au futur sophiste d'entrer en contact avec les milieux de Rome et de côtoyer le cercle des Flaviens.

Pour conclure sur ce point, Arrien a dû fréquenter le gymnase de Nicomédie, puis il a dû aller, comme tant d'autres jeunes gens de bonne famille, parfaire son éducation à l'étranger. Il est utile de remettre ces voyages dans leur contexte, en les comparant avec ceux d'un autre étudiant ou ami des lettres, $\varphi \imath \lambda o ́ \lambda o \gamma o \varsigma$, originaire de Nicomédie. Prousès, fils de Ménékratès, décéda à 24 ans à Mégare, au $\mathrm{I}^{\mathrm{er}}$ ou au $\mathrm{II}^{\mathrm{e}}$ siècle de notre ère ${ }^{50}$. L'iconographie de sa stèle funéraire est particulièrement suggestive: en-dessous du texte sont figurés deux rouleaux de papyrus (volumina) reliés et une boîte avec couvercle et poignée (capsula). Or, le choix de Mégare par ce jeune ami des lettres ne nous paraît pas anodin: en effet, Mégare (ou sa colonie Chalcédoine) avait été la métropole d'Astakos, fondée sur la rive sud du golfe d'İzmit/Nicomédie; la cité fut détruite par Lysimaque avant 281, alors que ses habitants furent relogés vers 264 av. J.-C. par le roi bithynien Nicomède I ${ }^{\text {er }}$ dans sa nouvelle capitale éponyme ${ }^{51}$, sur la rive nord du golfe. Dans cet endroit devait se trouver une autre colonie mégarienne, $\mathrm{Olbia}^{52}$. La nouvelle fondation s'appropria le passé hellénique, d'où l'appellation poétique donnée à Nicomédie, présente souvent dans les épigrammes

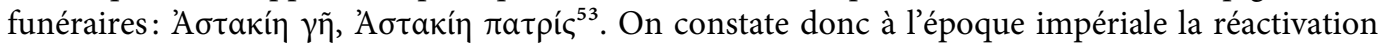
des liens de parenté, réelle ou légendaire. Pour rester dans le cadre bithynien, citons le dossier de la parenté entre Bithynion/Claudiopolis (nom dynastique), petite cité connue comme «Mantinée de Bithynie», et l'ancienne Mantinée d'Arcadie: il s'agit d'une parenté opportunément réactivée à l'occasion de l'essor du culte d'Antinoos, originaire de Bithynion ${ }^{54}$.

La formation d'Arrien, dans sa patrie, en Grèce continentale et dans un cadre panhellénique qui se renforce durant ces décennies, nous amène à nous interroger de plus près sur les origines et la carrière locale d'Arrien.

(47) Cf. Lucien, Alex. 2: «Arrien, disciple d'Épictète, homme appartenant à l'élite des Romains, qui consacra toute sa

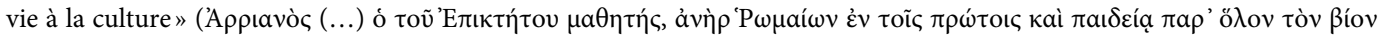
$\sigma v \gamma \gamma \varepsilon v o ́ \mu \varepsilon v o \varsigma)$ («Romain de la plus haute société, qui consacra toute sa vie aux belles lettres» M. Caster).

(48) Voir ci-dessous; de sa famille, on ne connaît que son cousin, et sans doute une autre branche (cf. la discussion sur Fl. Vlp. Arrianus).

(49) Desideri 1994; Fernoux 2004, p. 498-499; BekKer-Nielsen 2008.

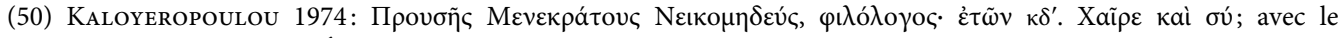
commentaire de L. Robert, $B E ́$, 1976, 289; une autre photo dans Delivorrias 2000, p. 56. Le nom du jeune défunt,

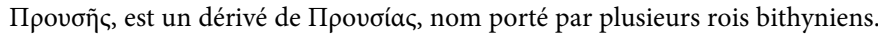

(51) Strabon 12.4.2 (C. 563); Pausanias 5.12.7.

(52) Selon Arrien, Bith. F 5 (= F 26 J.), Astakos fut appelée d'après un héros éponyme, fils de Poséidon et de la nymphe Olbia.

(53) Voir Robert 1939, p. 167-170 (=OMS, II, p. 1320-1323); Avram 2004, p. 977-978, nº 737; Fernoux 2004, p. 36-37.

(54) Voir Robert 1980, p. 132-146 («Mantinée de Bithynie. De la patrie d'Antinoos aux couvents byzantins»). 


\section{UN NOTABLE DANS SA PATRIE}

Né citoyen romain, dans une famille appartenant à l'aristocratie provinciale, Arrien était sans doute chevalier, accédant par la richesse de sa famille à l'ordre équestre ${ }^{55}$, avant d'entamer une carrière sénatoriale au début du règne d'Hadrien ${ }^{56}$.

Consul suffect, gouverneur d'au moins deux provinces, archonte à Athènes et «Nouveau Xénophon», tel fut le parcours de celui qui, à ses débuts, s'estimait fier d'avoir exercé à Nicomédie la prêtrise de Déméter et de sa fille Coré (F 1 de ses Bithyniaques) : «il a exercé, dit-il, la charge de prêtre de Déméter et de sa fille dans cette cité qui leur était consacrée ${ }^{57}$. Nicomédie était encore

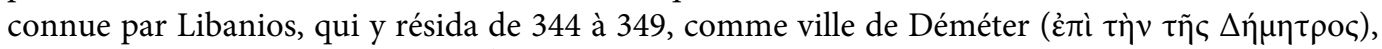
passage expliqué dans la scholie: «À savoir, Nicomédie: car là-bas le culte de Déméter est tenu dans la plus haute estime ${ }^{58}$. S'ajoute la précision topographique d'un texte hagiographique du $\mathrm{x}^{\mathrm{e}}$ siècle: «Carle plus grand sanctuaire de Déméter, qui est la déesse principale chez les Nicomédéens, était érigé près du théâtre ${ }^{59}$. C'est à Nicomédie, cité néocore sous Auguste et siège du koinon des

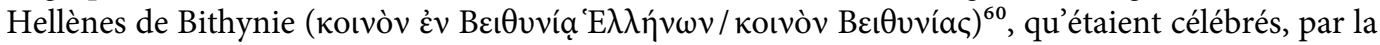
moitié occidentale de la province de Pont-Bithynie, les grands mystères de Déméter ${ }^{61}$.

Outre cette charge religieuse, la plus haute des prêtrises de sa cité, Arrien a dû assumer d'autres magistratures. Dans la seconde préface à l'Anabase, il précise, lors d'une présentation particulièrement construite: "Qui que je sois pour porter ce jugement sur moi-même, je n'ai aucun besoin d'inscrire mon nom, car il est loin d'être ignoré des hommes, ni de dire quelle est ma patrie, ma famille, ni les magistratures que j'ai pu exercer dans mon pays; il me suffit de dire que mes ouvrages sont et ont été, depuis ma jeunesse, ma patrie, ma famille et mes magistratures»

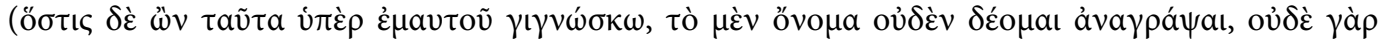

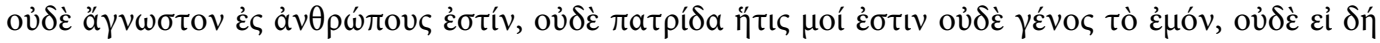

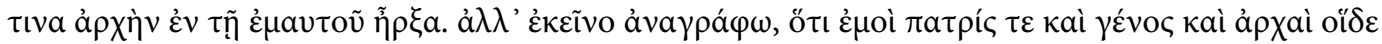

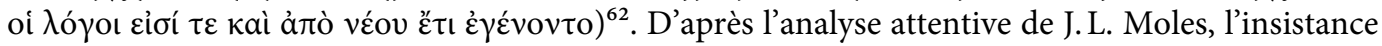

(55) Fernoux 2004, p. 423-425, nº 7.

(56) PIR F 219; Fernoux 2004, p. 456-458, n³2. Pour une reconsidération de sa carrière, nous renvoyons à l'étude d'Antony Hostein.

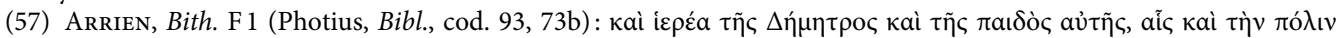

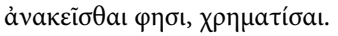

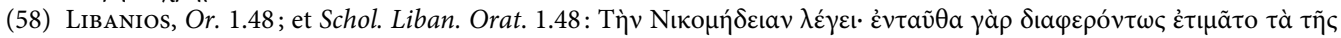

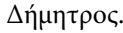

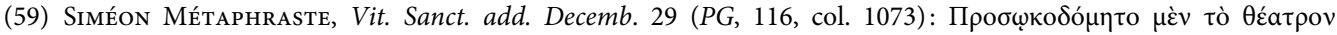

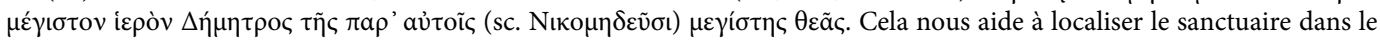
quartier Orhan Mahallesi d'İzmit, à proximité du théâtre (Boulhol 1994, p. 956-963). C’est d'ici que provient par ailleurs une dédicace en latin, sous Élagabal, Deae sanc(tae) Demetri (ŞAHIN 1974, p. 28-29, n 3 = TAM IV.1 54).

(60) Burrell 2004, p. 147-162; Guerber 2009, p. 83-84; Vitale 2012, p. 186-196.

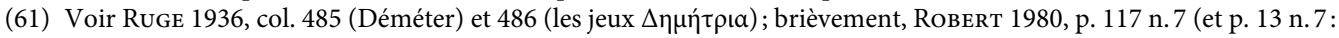
«Je traiterai ailleurs de ces mystères à Nicomédie»); Fernoux 2004, p. 66, sur la tribu «sacrée» de Nicomédie, Irpá, peutêtre consacrée à Déméter (cf. L. RoBERT, $B E, 1974,573$ ). Déméter est figurée sur des monnaies de Nicomédie sous plusieurs

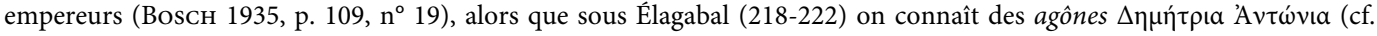
WADDINGTON $\left.1908, \mathrm{n}^{\circ} 281,282,250\right)$; son nom fut donné au $12^{\mathrm{e}}$ mois du calendrier bithynien $(\Delta \eta \mu \eta ́ \tau \rho ı \varsigma)$.

(62) Anab. 1.12.5 (trad. P. Savinel modifiée). Ce texte a été abondamment commenté: BoswORTH 1972, p.174-175; Moles 1985; Marincola 1989 (en particulier p. 188-189); Swain 1996, p. 244-245 (le modèle homérique, y compris pour l'anonymat); Rodríguez Horrillo 2011. Gray 1990 surprend la manipulation de quatre topoi rhétoriques: (1) les détails personnels (nom, famille, pays, carrière pour mesurer la valeur); (2) les motifs importants pour la vie d'une personne (nom, famille, pays, magistratures); (3) l'équation de la réalisation littéraire et pratique; (4) la prétention à la suprématie dans un domaine. 
sur l'anonymat et la recusatio de son nom, de son pays et de ses magistratures, sont délibérées et ostentatoires $^{63}$.

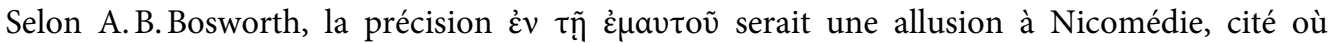
il fut peut-être archonte, agoranome et agonothète ${ }^{64}$. Il suit en cela W. Ameling: Arrien a dû être archonte de sa cité, $\pi \rho \tilde{\omega} \tau o \varsigma$ ä $\rho \chi \omega v$, magistrature liée à celle d'agonothète, puisque les deux déesses sont en rapport avec le culte impérial ${ }^{65}$; de cette manière, observe Ameling, le lien d'Arrien avec sa patrie semble avoir été plus fort que celui de Dion Cassius, qui ne semble pas avoir exercé de magistrature à Nicée ${ }^{66}$. Pour ne citer qu'un seul parallèle dans la région, le sophiste Memmios Markos de Byzance est, grâce aux mentions numismatiques ${ }^{67}$, connu pour avoir assumé la charge de hiéromnamôn (magistrat éponyme) de sa cité au milieu du $\mathrm{II}^{\mathrm{e}}$ siècle.

À quel moment peut-on dater cette carrière locale d'Arrien? Comme le suppose, entre autres, Bernard Rémy, Arrien rentra sans doute dans sa patrie pour accomplir des liturgies et des charges, entre 117 et $120 / 121^{68}$, avant de se consacrer à une carrière sénatoriale tout au long du règne d'Hadrien.

On ignore presque tout des éventuels honneurs octroyés à Arrien ou bien du profit que sa patrie n'a pas tardé à en tirer, en se vantant de son ressortissant le plus célèbre sous le philhellène Hadrien. Pour l'instant, on ne dispose que d'un seul exemple d'hommage civique pour Arrien ${ }^{69}:$ il s'agit d'un décret honorifique, érigé par ailleurs sur la proposition de son propre cousin, P. Aelius Poléméanos Hermodôros, conformément à la décision du conseil et de l'assemblée de Nicomédie:

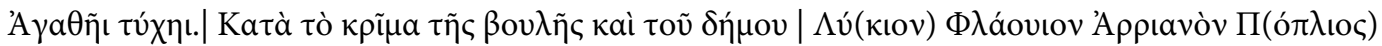

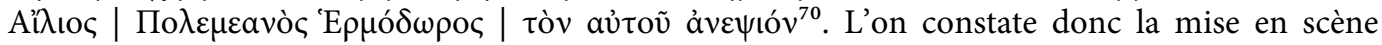
des liens familiaux, où le proposant du décret est identifié par rapport à un parent célèbre. Le gentilice d'Hermodôros nous aide à dater l'inscription après 117, vraisemblablement au cours de la quatrième décennie du $\mathrm{II}^{\mathrm{e}}$ siècle: Arrien, qui, au moment de la décision, devait être légat de Cappadoce, avait très probablement facilité par une intervention auprès de l'empereur l'accès à la citoyenneté romaine de son cousin. Le peu que l'on peut dire sur le cousin d'Arrien est qu'il s'agit d'un citoyen nouveau, connu auparavant comme Hermodôros, sans doute fils de Polémaios ${ }^{71}$.

À l'exception de cette précieuse attestation, nous ignorons tout de la mémoire que Nicomédie a dû cultiver de son fils le plus célèbre, en attendant d'autres découvertes épigraphiques. Ainsi, une possible branche de la famille vient d'être attestée dans une inscription honorifique posthume,

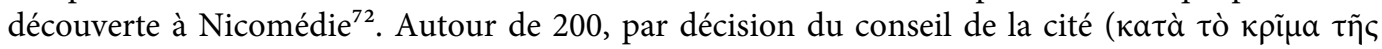

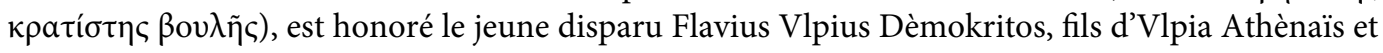
de Flavius Vlpius Arrianos. Ce dernier avait été un notable de premier rang, puisque sa carrière est présentée en détail, alors que son fils n'eut pas l'occasion de s'affirmer. Le père avait été prêtre (de

(63) Topos qui reviendra, par d'autres moyens, sous la plume de penseurs tels CAmus, «Oui, j’ai une patrie: la langue française" (Carnets II, janvier 1942-mars 1951, Paris, 1964, p. 337) et CIORAN, "On n'habite pas un pays, on habite une langue. Une patrie, c'est cela et rien d'autre» (Aveux et anathèmes, Paris, 1987, p. 21).

(64) BoswOrTH 1980, p. 106; contre WIRTH 1964, p. 224, qui pense à une allusion à l'archontat athénien et 1988, p. 35-36).

(65) Pour cette magistrature, voir Ameling 1984 b (en partic. p. 24).

(66) Ameling 1984c, p. 129-130.

(67) Voir Dana 2011, p. 253-254.

(68) RÉMY 1989, p. 213-217, nº 169.

(69) TAM IV.1 402, sur Arrien gouverneur de Cappadoce, consul et prêtre de Déméter et Perséphone, est un faux (voir, entre autres, F. JACOBY, FGrHist, II.B. Komm., p. 553).

(70) SEG I 446a = TAM IV.1 41a.

(71) Pour ce type d'indication, voir Consten 2010. Dörner transcrit Пo $\ell \varepsilon \mu \varepsilon<1>a v o ́ \varsigma$; LGPN V.A 370 donne la forme

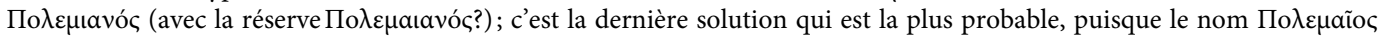
est attesté par trois fois en Bithynie (LGPN V.A 370).

(72) Adak, Stauner 2013. 
Déméter ou du culte impérial?), distributeur d'argent au conseil et à l'assemblée, premier archonte, censeur, curator à Pruse de l'Olympe, organisateur d'une parapompè.

Nous pouvons même aller plus loin: le "Nouveau Xénophon", installé à Athènes, inscrit dans le dème de Paiania et honoré en tant qu'archonte éponyme en $145 / 146^{73}$, ne coupa pas les liens avec sa région d'origine. En effet, un décret de l'année de son archontat fait l'éloge du cosmète Athénaios, fils d'Alexandros, de Rhamnonte, comme hiéronique et xystarque à vie en Bithynie ([ $\xi_{v} \sigma \tau \alpha \dot{\alpha} \rho \chi \eta v$

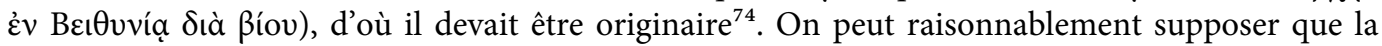
détention de cette magistrature pouvait être due à l'exercice de l'archontat par Arrien, célèbre Bithynien établi à Athènes ${ }^{75}$.

Le lien le plus profond avec sa patrie reste néanmoins l'histoire locale qu'Arrien a rédigée en guise de don à sa chère Nicomédie. Ce sera le dernier volet de notre étude, celui qui nous permettra de nous rendre compte non tant du talent d'historien d'Arrien - son œuvre n'est connue qu'à travers le résumé du Photius et quelques fragments - mais de son intérêt profond et réel pour les antiquités de son pays.

\section{LES BITHYNIAQUES, ÉLABORATION D'UNE HISTOIRE DE LA BITHYNIE}

Parmi les ouvrages d'Arrien lus et résumés ${ }^{76}$ par le patriarche byzantin Photius, dans la seconde moitié du IX ${ }^{e}$ siècle, se trouvent ses Bithyniaques (cod. 93), en 8 livres ${ }^{77}$ :

$\mathrm{Lu}$ du même auteur l'Histoire de Bithynie, en huit livres, où il rapporte en détail les légendes de Bithynie et les autres faits qui la concernent; il fait don à sa patrie du récit de ses antiquités ( $\tau \tilde{n}$

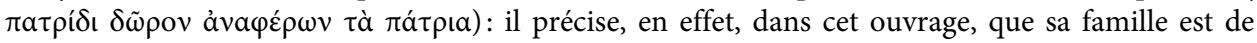
Nicomédie et que c'est dans cette ville qu'il est né, qu'il a été élevé et instruit. Il a exercé, dit-il, la charge de prêtre de Déméter et de sa fille dans cette cité qui leur était consacrée.

La préface de cette histoire locale présente, comme l'observe J.M. Marincola, un ensemble différent de conventions par rapport à la seconde préface de l'Anabase ${ }^{78}$ : Arrien insiste à cette occasion sur son origine de Nicomédie, y compris sur le fait d'y avoir vécu et d'y avoir été instruit. Il y il avait assumé la plus haute magistrature religieuse, sans oublier de préciser que l'histoire des

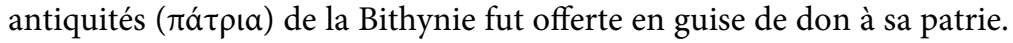

En réalité, plutôt que d'un résumé, il s'agit d'une présentation générale et d'une exposition de la préface. Dans le codex 58, Photius avait déjà brièvement présenté les Bithyniaques ${ }^{79}$ : «(Arrien)

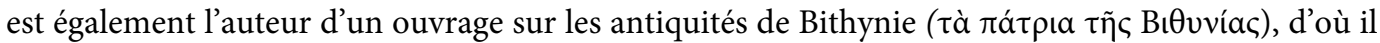

(73) Follet 1976, p. 34-36, 209-212.

(74) $I G \mathrm{II}^{2}$ 3741. Dans l'inscription $I G \mathrm{II}^{2}$ 2055, on retrouve l'archonte Arrien (de Paiania) et le cosmète Athénaios; ce dernier est sans doute mentionné dans $I G \mathrm{II}^{2} 3145$ : [...] fils d'Alexandros, de Rhamnonte, victorieux dans plusieurs concours.

(75) Voir Follet 1976, p. 209 et 212; alors que d'autres savants en font le xystarque de toute de la province de Bithynie, Robert 1966, p. 82 n. 5, préfère y voir «l'indication vague d'une xystarchie dans une ville de Bithynie».

(76) D'autres ouvrages: les Parthika en 17 livres; l'Histoire après Alexandre en 10 livres; l'Alanikè; les biographies de Dion, de Timoléon et du brigand Tillorobos.

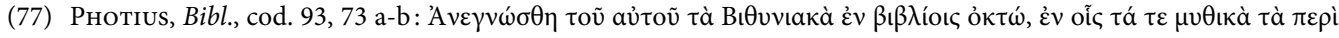

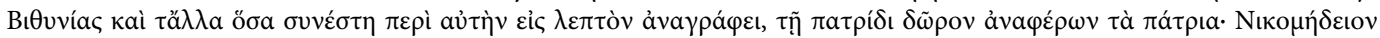

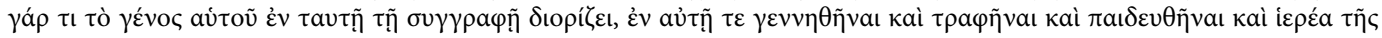

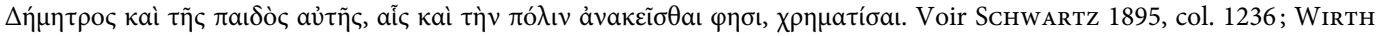
1974, p. 194; STADTER 1980, p. 152-161; BOSWORTH 1988, p. 28-29; TONNET 1988, I, p. 11-17.

(78) Marincola 1989, p. 188.

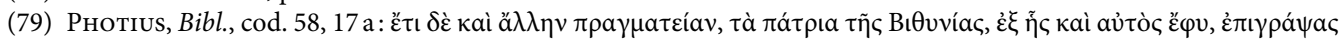

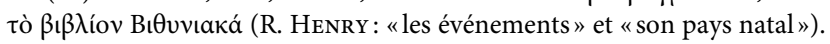


était issu lui-même; il a intitulé le livre Histoire de Bithynie». Or, c'est la graphie même du titre qui pose problème: les fragments conservés présentent en effet deux formes, Bıtuvıká (forme préférée par A.G. Roos), ou plutôt Bı $\theta v v ı \alpha^{8}{ }^{80}$ (forme préférée par Ph. A. Stadter).

D'autres fragments ${ }^{81}$, de longueur et d'origine variées (extraits, notices, échos) sont connus essentiellement par Étienne de Byzance (nota bene, au vi ${ }^{\mathrm{e}}$ siècle, dans la version abrégée de ses 'EOvıкá), par l'érudit Eustathe de Salonique (ca. 1175), dans ses commentaires d'Homère et de Denys le Périégète (Anaplus Bospori), et par le philologue Jean Tzetzès, au XII ${ }^{\mathrm{e}}$ siècle. Il importe donc avant tout de prendre en compte l'intérêt occasionnel des érudits et des compilateurs byzantins, ce qui explique le caractère déconcertant des fragments conservés, majoritairement d'intérêt mythologique et géographique.

Comprendre les intentions d'Arrien nécessite d'abord de connaître la date ${ }^{82}$ de composition de son histoire locale. Or, force est de constater que, pour l'ensemble de l'œuvre d'Arrien ${ }^{83}$, seuls quatre ouvrages tardifs sont bien datés: le Périple du Pont-Euxin, après 131; la Tactique, vers 136/137; l'Ordre de marche contre les Alains, après 135; le Cynégétique, dans les années 140. Qui plus est, même la date de l'Anabase est disputée, et souvent les spéculations partent de la supposition que, vers la fin de sa vie, plus précisément après 137, Arrien se fixa à Athènes. Plusieurs commentateurs ont préféré voir dans les Bithyniaques, sur la foi de Photius, un ouvrage tardif ou de maturité:

Il rappelle dans le présent livre d'autres œuvres; l'une d'elles rapporte les faits et gestes du Corinthien Timoléon en Sicile, une autre les actes dignes de mémoire accomplis par Dion de Syracuse quand il délivra cette ville avec toute la Sicile de Denys II, fils de Denys Ier, et des Barbares que Denys avait amenés pour exercer sa tyrannie en sécurité. Il est évident que cette histoire de sa patrie est le quatrième ouvrage qu'il a écriit ${ }^{84}$, car c'est après les histoires d'Alexandre, de Timoléon et de Dion que ce traité a été composé. C'est d'emblée, dès qu'il fut capable d'écrire, qu'il avait voulu mettre sur pied ce sujet et le traiter, mais le travail préparatoire nécessité par son information incomplète avait allongé les délais. Telle est, en effet, la raison qu'il donne lui-même de sa lenteur ${ }^{85}$.

Le souhait d'écrire une histoire locale, tout comme l'insistance sur le travail de documentation, représentent à la fois des lieux communs et une affirmation indirecte de l'importance de son entreprise.

Or, la datation est capitale: le fait d'écrire cette histoire locale avant de connaitre la célébrité, ou de l'écrire après l'avoir acquise, est tout sauf anodin. Cela oblige à s'interroger sur l'intention, la vision, enfin le caractère conférés par Arrien à ses Bithyniaques. Pour les partisans d'un ouvrage tardif, tel Henri Tonnet ${ }^{86}$, il s'agirait d'un souvenir reconnaissant de sa province natale, où il montre les prétentions de sa patrie à une origine grecque. Dans la même ligne, Bosworth date l'Anabase entre 115 et 125, et place les Bithyniaques peu après cette période ${ }^{87}$. En revanche, Stadter

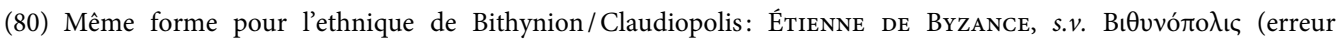

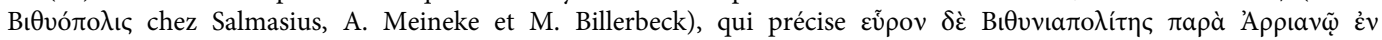
$\varepsilon^{\prime}$ Bı $\theta v v \iota \alpha \kappa \tilde{\omega} v$ (Bith.F $7=$ F. 17 J.).

(81) Les fragments sont rassemblés par Felix Jacoby (FGrHist 156 F 14-29; commentaire: F. JACOBY, FGrHist II.B, nº 156, p. 564-566) et par A. G. Roos et G. WIRTH (Flavii Arriani quae extant omnia, II. Scripta minora et fragmenta, Leipzig, Teubner, 1967, p. 198-223), qui ont rassemblé 72 fragments; un autre possible fragment a été identifié par MerKELBACH 1985. Aucun fragment n'est transmis par la Souda.

(82) Wirth 1964; Bosworth 1972, p. 179-181; StADTER 1980, p. 179-187.

(83) Pour la chronologie des ouvrages d'Arrien, voir TonNET 1988, I, p. 60-99 et 101.

(84) Après l'Histoire d'Alexandre en 7 livres (cod. 91, 67b-68b), Histoire des événements postérieurs à Alexandre en 10 livres (cod. 92, 69a-73), les biographies de Timoléon et de Dion.

(85) Pнотіus, Bibl., cod. 93, 73 b.

(86) F. JAсовy, FGrHist, II.B. Komm., p. 552; Tonnet 1988, I, p. 69 et 554 (l'Anabase entre 137 et 165 ; les Bithyniaques après l'Anabase).

(87) Bosworth 1988, p. 28-29 (les biographies de Dion et de Timoléon avant les Bithyniaques, car elles sont mentionnées dans la préface de cet ouvrage). 
date l'Anabase après le consulat d'Arrien et, avec raison, les biographies de Dion et de Timoléon ${ }^{88}$ avant les Bithyniaques, qu'il estime être un ouvrage de jeunesse, rédigé peut-être dans les années 120 , en parallèle avec les débuts de sa carrière sénatoriale ${ }^{89}$. Comme certains commentateurs, nous penchons pour une datation après les guerres parthiques - qui ont occasionné la rédaction des Parthiques -, mais avant qu'Arrien ne s'engage dans sa carrière sénatoriale, au début des années $120^{90}$.

Précisons également le contexte littéraire. Ce type d'ouvrage s'inscrit dans le genre très prolifique des histoires locales (Lokalgeschichte, selon l'expression consacrée par Felix Jacoby) ou des monographies régionales, et beaucoup moins dans la tradition de la littérature gréco-juive, gréco-égyptienne ou gréco-phénicienne, plus soucieuse de la mise en valeur d'un passé différent, voire antérieur à celui des Grecs. Le nombre des fragments sur la Bithynie, ancien royaume indigène hellénisé au Nord-Ouest de l'Asie Mineure, habité par une population thrace distincte, est assez élevé, et cela n'est pas sans rapport avec le dynamisme culturel de la région ${ }^{91}$. Deux autres lettrés de Bithynie sont connus comme auteurs de Bithyniaka, sans doute en dix livres: au I ${ }^{\text {er }}$ siècle av. J.-C., le médecin Asclépiade de Myrleia (= Apamée de Bithynie) (FGrHist 697) et, à une date inconnue, un certain Démosthénès (FGrHist 699 ${ }^{92}$. Il faut néanmoins reconnaître que la plupart des fragments conservés des trois Bithyni(a)ka proviennent de l'ouvrage d'Arrien (72 numéros dans l'édition de Roos) : ce choix fut déterminé plutôt par sa célébrité, que par la valeur intrinsèque des notices. Pour donner un autre exemple dans la région, plusieurs histoires locales concernent Héraclée du Pont, où cette discipline fut illustrée notamment par Memnon, résumé par le même Photius ${ }^{93}$.

En plus des commentaires ponctuels de Felix Jacoby, la meilleure analyse des Bithyniaques fut donnée par Philip A. Stadter: il s'agit d'une description de son pays, sans doute centrée sur le

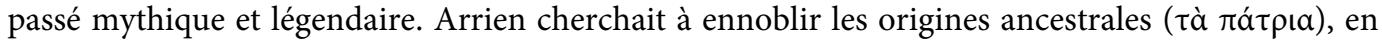
démontrant que les Thraces bithyniens, loin d'être des barbares sauvages, selon l'image consacrée par toute une tradition littéraire grecque, étaient apparentés aux héros de la mythologie grecque: arrivés d'Europe, ils avaient apporté la civilisation en $\mathrm{Asie}^{94}$.

Il convient également d'évoquer les difficultésinhérentes du sujet. La Bithynie était une région initialement habitée par une population non grecque ${ }^{95}$, avec un nombre réduit de colonies grecques (notamment Chalcédoine et Astakos) et plusieurs fondations ou refondations dynastiques (Nicomédie, Pruse de l'Olympe, Pruse de l'Hypios, Bithynion, etc.), par une maison royale

(88) Biographies de Dion, de Timoléon (général corinthien) et du brigand Tillorobos (FGrHistCont 1069).

(89) Chronologie de Stadter: Bithyniaka, Parthika, Anabasis (et Indikè), Histoire des événements postérieurs à Alexandre.

(90) Voire in absentia, suggestion de Madsen, p. 69.

(91) Sur les histoires locales de la Bithynie, voir Meyer 1897, col. 524; Aréthas, évêque de Césarée de Cappadoce (IX ${ }^{\mathrm{e}}-\mathrm{X}^{\mathrm{e}}$

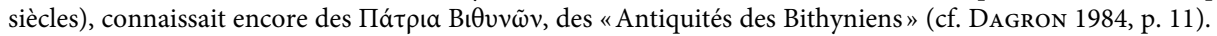

(92) Alors que le polygraphe Alexandre Polyhistor de Milet (ca. 80-35 av. J.-C.) (FGrHist 273 F 12-13) et Artémidore

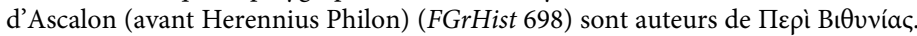

(93) Voir Dana 2011, p. 246.

(94) Arrien, Bith. F 19 (les Thraces de Pataros chassent d'Asie les envahisseurs cimmériens); voir F. JAcoBy, FGrHist, II.B. Komm., p. 564 (sur le lien étroit avec le monde mythique hellénique), et WIRTH 1974, p. 194 (sur l'appartenance de la Bithynie à la culture grecque).

(95) Les Bithyniens, une population thracophone, appelés «Thraces d’Asie» par HÉrodote (3.90 et 7.75) et XéNOPHON (Anab. 6.4.2), ou les "Thraces bithyniens» (Hérodote 1.129; Thucydide 4.75; Xénophon, Hell. 1.3.2 et 3.2.2), avec un écho archaïsant choisi par Arrien (Anab. Alex. 1.29.5, sur la Thrace de Bithynie); cf. aussi Arrien, Bith. F 19. Or, le modèle révéré d'Arrien, Xénophon, avait noté leur l'hostilité foncière à l'égard des Grecs (Anab. 6.4.2): «(Entre Byzance et Héraclée) il n'y a aucune cité qui soit amie ou grecque mais ce sont les Thraces bithyniens. On dit qu'ils font subir les pires violences aux Grecs quand ils les prennent, qu'ils soient naufragés ou d'une autre façon»); c'était un détail embarrassant aux yeux d'Arrien, qui modifie le texte de Xénophon: «les Thraces bithyniens, dont Xénophon aussi a fait mention dans son ouvrage [l'Anabase], disant qu'ils sont les plus belliqueux en Asie» (ARrien, PPE 13.6); de même, si les épaves sont évoquées à propos de Salmydessos, le pillage des Thraces est omis (ARRIEN, PPE 25.2), comme ne manque pas de remarquer ToNNET 1988, I, p. 12. 
d'origine indigène, bien que profondément hellénisée ${ }^{96}$. L'absence de toute mention homérique poussa Arrien à en trouver des allusions dans divers passages, puisque l'ancrage homérique était essentiel à ses yeux, comme pour Strabon.

Les quelques 70 fragments et échos permettent néanmoins de se faire une idée générale de cet ouvrage perdu. Les moyens employés sont variés: la généalogie mythologique, l'intégration de l'histoire locale dans l'histoire générale grecque, l'historicisation des événements mythiques, le recours aux héros éponymes, même s'il s'agissait plutôt de faire entrer les éponymes (inventés) des autres peuples dans les généalogies grecques ${ }^{97}$. On remarque également l'abondance d'étymologies et d'étiologies, puisqu'Arrien y abordait, entre autres, le thème de l'origine des Bithyniens, en décrivant parfois leurs croyances. De cette manière, le rôle de la Bithynie avant l'arrivée de Rome apparaît plutôt comme un produit des lectures érudites d'Arrien ${ }^{98}$, dans le cadre exclusif de la mythologie grecque, où il apparaît en fin connaisseur d'Homère et de la rhétorique.

Malheureusement, le caractère particulier des fragments conservés nous empêche de mieux saisir sa vision de la Bithynie, d'autant plus que, selon Henri Tonnet, «la façon dont il a imaginé le passé de sa province est révélatrice de la manière dont il conçoit son identité »" ${ }^{99}$ On constate toutefois que, dans cette histoire locale, il faut prendre en considération trois échelles distinctes, où évoluent trois acteurs différents mais en constante interaction: le plan bithynien, le cadre général du monde hellénique - y compris par les références répétées au passé mythologique hellénique -, et l'arrivée du pouvoir romain. On se situe à tout moment, dès les origines mythiques, dans un cadre universel: cela est particulièrement manifeste chez Memnon, l'auteur d'une monographie sur Héraclée du Pont, du moins d'après le résumé de Photius.

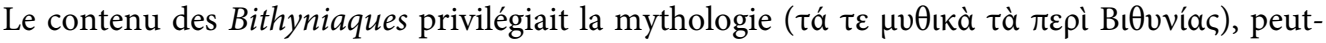

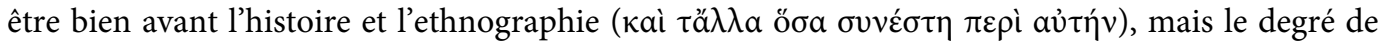
perméabilité entre les trois domaines d'interaction nous reste inaccessible. De prime abord, les références mythologiques abondent, du moins dans les fragments conservés. L'ancêtre éponyme des Bithyniens serait Bithynos, fils de Zeus et de la nymphe Thraké ${ }^{100}$; de cette façon, les descendances généalogiques grecque et thrace sont étroitement, même indistinctement, associées. On en trouve

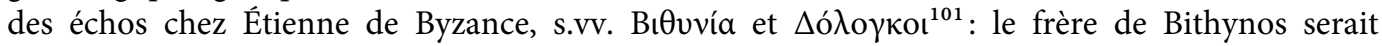
Dolonkos (éponyme des Thraces Dolonkoi, sur le continent européen), fils de Cronos et de Thraké.

Arrien fournissait une autre généalogie éponymique, d'après laquelle Thynos et Bithynos seraient les fils d'Odrysès, adoptés par Phineus, père de Paphlagon ${ }^{102}$. Ce scénario servait encore

(96) Voir l'étude stimulante de Hannestad 1996.

(97) Pour un débat récent sur les «spécificités locales» qui ne font que mieux participer à la "globalisation» du monde gréco-romain, voir Withmarsh 2010; VLassopoulos 2011 et 2013 (p. 226-277).

(98) Selon l'observation pertinente de STAdTer 1980, p. 154. Le même procédé est manifeste dans son Périple du Pont-Euxini, occasionné par son voyage d'inspection en 131, entre Trapézonte et Dioscurias, en qualité de gouverneur de Cappadoce: caractère littéraire marqué, abondance des références culturelles (mythologiques, historiques, voire archéologiques, cf. SiLBERMAN 1993).

(99) Tonnet 1988, I, p. 11-17.

(100) Cf. Appien, Mithr. 1.2: selon d'autres auteurs, Bithys, fils de Zeus et de Thraké, avait été le premier roi des

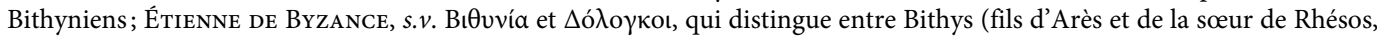
s.v. Bitúal) et Bithynos (fils de Zeus et de Thraké). Sur cette légende, voir RoBert 1980, p. 131.

(101) Étienne De Byzance, s.v. Bıtvvía: «pays près du Pont, qui tire son nom de Bithynos et de la Titanide Thraké,

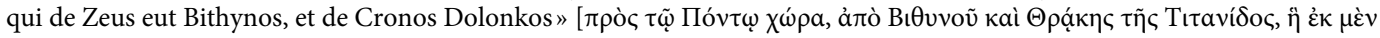

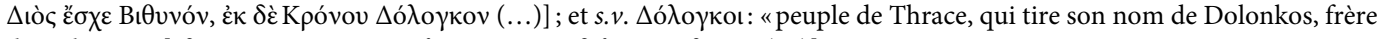

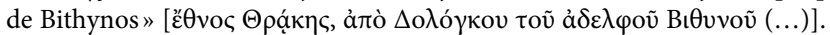

(102) Arrien, Bith. F20 (=F78 J.) (Eustathe De Salonique, Ad Dionys. 793): «Selon lui-même Arrien, on raconte que

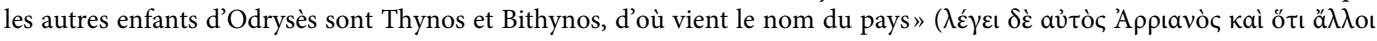

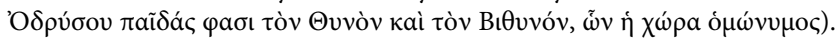


mieux à concilier descendance thrace (par le père Odrysès et par le frère Thynos) et nouvelle patrie en Asie Mineure, dans le voisinage de la Paphlagonie ${ }^{103}$.

Il s'agit certes d'un usage particulièrement répandu à l'époque, avec l'exhibition, sur le monnayage provincial, des héros éponymes, souvent inventés, attestant l'ancienneté et l'origine illustre de chaque cité. On peut citer à ce propos, chez son contemporain Appien, un passage plus développé sur les origines mythiques des Illyriens ${ }^{104}$ :

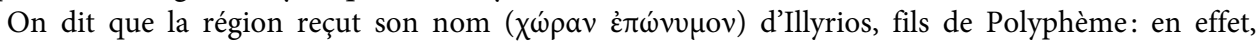
Polyphème le Cyclope et Galatée eurent des fils, Celtos, Illyrios et Galas, qui partirent de Sicile et commandèrent à ceux que l'on appelle, à cause d'eux, Celtes, Illyriens, Galates. Bien que beaucoup

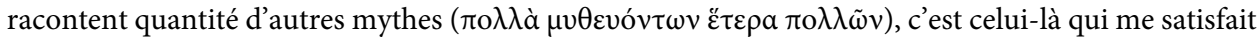
le plus. [4] Illyrios eut pour fils Enchéleus, Autarieus, Dardanos, Maedos, Taulas et Perrhaebos, et pour filles Parthô, Daorthô, Dassarô et d'autres, dont sont issus les Taulantiens, les Perrhèbes, les Enchéléens, les Autariates, les Dardaniens, les Maedes, les Parthènes, les Dassarètes et les $\mathrm{Da}<0>$ rses. On estime que d'Autarieus naquit un fils, Pannonios ou Paeôn, et de Paeôn Scordiscos et Triballos,

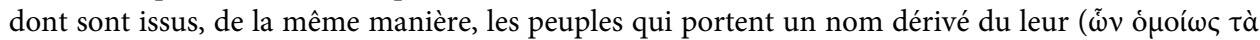

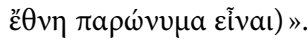

Pour ce qui est du contenu historique, qui devait être fortement imbriqué au plan mythologique, on connaît au moins la limite chronologique. Les Bithyniaques traitaient de l'histoire de la Bithynie jusqu'à la fin de l'indépendance, selon l'usage ${ }^{105}$ : «Il commence donc son récit, comme on l'a dit,

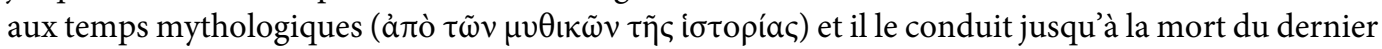
Nicomède, qui, en mourant, laissa par testament son royaume aux Romains, qui n'avaient plus de rois depuis l'expulsion de Tarquin ${ }^{106}$. C'est en effet un point remarquable: Arrien achève son histoire en 74 av. J.-C., à la mort de Nicomède IV Philopator, qui légua son royaume aux Romains. Arrien devait aussi gommer un peu la collaboration de ses compatriotes avec Mithridate VI, lors de cette période trouble.

Il convient toutefois de remarquer qu'Arrien n'écrit pas l'histoire de la seule Nicomédie ${ }^{107}$, mais il va de soi que sa propre cité devait y avoir une place de choix ${ }^{108}$. Cela n'était pas pour lui déplaire et pas difficile non plus à illustrer: l'ancienne capitale royale qui garda son nom dynastique fut par la suite le centre d'un koinon des cités libres de Bithynie et des onze politeiai du Pont. C'était un capital précieux dans le contexte de la concurrence acerbe avec sa voisine Nicée pour le titre de métropole de Bithynie - titre détenu très tôt par Nicomédie, et presque sans partage.

Or, il se trouve que, sur l'histoire proprement-dite du royaume - Arrien a écrit huit livres -, on ne possède que très peu de fragments (F 63-64), insignifiants et déformés: par exemple, l'anecdote transmise par Jean Tzetzès, dans ses Chiliades, sur la mort tragique de la reine Dintizila ${ }^{109}$, première

(103) Aux époques mythiques, le pays aurait été occupé par les Bébryces, point de raccord avec la légende des Argonautes (Bith. F 38), et par les Halizones d'Homère (Bith. F 22); un autre repère chronologique, à moitié légendaire, est fourni par l'invasion des Cimmériens (Bith. F 19 = Eustathe de Salonique, Ad Dionys. 322): «Arrien dit que les Thraces passèrent d'Europe en Asie avec leur chef, un certain Pataros, quand les Cimmériens faisaient leurs incursions en Asie; les Thraces les chassèrent de Bithynie et s'y installèrent». Ces derniers présentaient deux branches: les Thynes, au nord, sur les littoraux européen et asiatique de la Mer Noire; plus au sud, les Bithyniens, qui repoussèrent leurs cousins mysiens au-delà de l'Olympe mysien (Bith. F 21 = Eustathe de Salonique, Ad Dionys. 809).

(104) Appien, Illyr. 2.3-4 (trad. P. Goukowski).

(105) À l'instar, par exemple, de Memnon d'Héraclée.

(106) Photius, Bibl., cod. 93, 73 b.

(107) Un certain Ménékratès (d'époque hellénistique?) est l'auteur d'un ouvrage Пвpì Nıкaíac (FGrHist 701).

(108) Voir Bith. F 9: Arrien décrit le culte de Cybèle, qui avait un temple sur l'agora de Nicomédie et était figurée sur

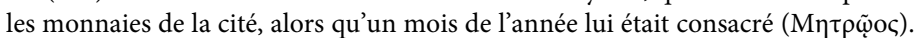

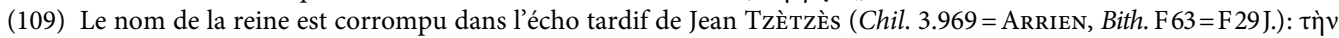

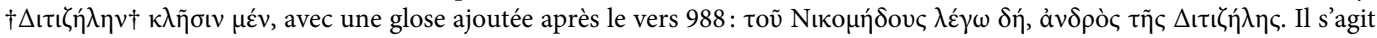

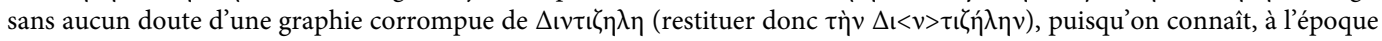


épouse de Nicomède I er (ca. 280-250 av. J.-C.), dont la tombe monumentale recouverte d'or, devenue un lieu de mémoire, se trouvait à Nicomédie ${ }^{110}$.

Enfin, il vaut la peine de mentionner quelques fragments d'intérêt ethnographique, telle une coutume lors des procès ${ }^{111}$ : «C'est pourquoi les Bithyniens, comme rapporte Arrien, rendaient la justice en s'asseyant au-devant du soleil, comme si le dieu les observait». Une autre coutume est la polygamie des Thraces, qui s'expliquerait, selon Arrien, par le but d'avoir beaucoup d'enfants. L'historien attribuait en effet cette pratique au roi Dolonkos, qui eut une nombreuse descendance de plusieurs femmes ${ }^{112}$.

Plutôt qu'un ouvrage nostalgique, qu'on aurait du mal à placer à une époque où les intérêts d'Arrien et les attentes de son public étaient plus larges, les Bithyniaques constituent un ouvrage où l'auteur, encore impliqué dans la vie de Nicomédie, rend hommage à sa patrie bithynienne. Il accomplit ainsi un projet de jeunesse, avant de se tourner vers d'autres horizons ${ }^{113}$.

Nous avons étudié ici "Arrien le Bithynien». Mais nous avons immédiatement remarqué que, dans son œuvre comme dans son parcours, les référents hellénique et romain sont indissociables de son origine provinciale. Cela montre qu'en fin de compte Arrien est un homme aux multiples patries, physiques et symboliques: sa première patris est Nicomédie, plus généralement la Bithynie, dont il décrit les antiquités dans ses Bithyniaques, en les inscrivant, à l'aide de nombreuses références mythologiques et littéraires, dans le passé hellénique. Plus connues, ou plus étudiées, sont l'appartenance hellénique, qui est indéniable, y compris par l'octroi de la citoyenneté athénienne à celui qui se revendiquait comme "Nouveau Xénophon», ainsi que l'adhésion sincère au pouvoir de Rome, sans aucune réticence ni résistance. Ces deux identités, loin de l'effacer, se sont au contraire ajoutées à son appartenance bithynienne, si toutefois elles ne le caractérisaient dès le début: depuis sa plus tendre enfance Arrien fut connaisseur d'Homère, et, depuis sa naissance, il fut citoyen de l'Empire, avant d'assumer de hautes responsabilités. À l'instar de ses héros, légendaires ou historiques, qui traversent l'espace géographique et chronologique d'un passé exemplaire, Arrien fut un connaisseur et un passeur entre plusieurs mondes. Enfin, une dernière patrie, la plus précieuse, celle qui devait lui assurer sa célébrité, est mise en avant par l'écrivain, qui tient ses écrits pour la marque de son excellence - alors qu'il avait acquis une notoriété par sa carrière au service de l'Empire. Pour Arrien, cette patrie, sorte de République des lettres, l'emportait en ancienneté et en prestige sur les autres: «il me suffit de dire que mes ouvrages sont et ont été, depuis mon enfance, ma patrie, ma famille et mes magistratures».

Dan DANA

CNRS -UMR 8210 ANHIMA

Madalina DANA

Université Paris 1 Panthéon-Sorbonne - UMR 8210 ANHIMA

impériale, deux occurrences de ce nom: $\Delta \mathrm{t} v \tau \iota \zeta \lambda \alpha$ dans le territoire de Pruse de l'Olympe (I.Prusa ad Olympum I 80, au $\mathrm{I}^{\mathrm{er}}$ siècle av. J.-C.); $\Delta \mathrm{v} \tau \iota_{\iota} \zeta_{\mathrm{I}}[\lambda \alpha]$ au Nord-Est de la Bithynie (SEG LII 1224, au II siècle av. J.-C.). Voir Cl. BRIXHE (BÉ, 1991, 576, explication assez confuse); Corsten 2006, p. 121-123; DANa 2006, p. 134.

(110) Cf. Fernoux 2004, p. 41 : Arrien a pu voir à Nicomédie la sépulture de la première épouse de Nicomède ${ }^{\text {er }}$ (Bith. F 63), ainsi que celle du roi Prusias, qui ne fut détruite qu'en 101 (Dion Chrysostome, Or. 47.16).

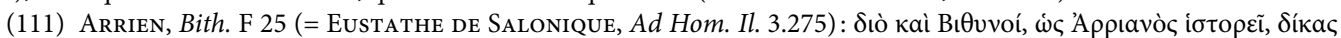

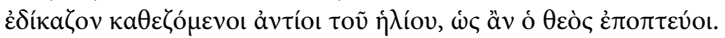

(112) Arrien, Bith. F 13 (= Eustathe de Salonique, Ad Dion. 322).

(113) StADTER 1980, p. 167: «The Bithyniaca may represent a more nostalgic moment». 


\section{Bibliographie}

Adak M., Stauner K., 2013, «Eine Honoratiorenfamilie aus Nikomedeia», Gephyra, 10, p. 143-151.

Aкyürek ŞAhIn E., 2011, «Nikomedeia'dan iki entellektüel: Basilikos ve Kyrion'un mezar epigramları », Olba, 19 , p. 349-370.

Ameling W., 1984a, «L. Flavius Arrianus Neos Xenophon», EA, 4, p. 119-122.

Ameling W., 1984b, «Die Archontat in Bithynien und die Lex Provinciae des Pompeius», EA, 3, p. 19-31.

Ameling W., 1984c, «Cassius Dion und Bithynien», EA, 4, p. 123-138.

Avram A., 2004, «The Proponic Coast of Asia Minor», dans M.H.Hansen, Th. H. Nielsen (éd.), An Inventory of Archaic and Classical Poleis, Oxford.

Avram A., 2013a, Prosopographia Ponti Euxini Externa, Louvain-Paris-Walpole (MA) (Colloquia Antiqua 8).

Avram A., 2013b, «Les Bithyniens en Thrace et en Mésie inférieure et dans le Pont Nord à l'époque impériale», à paraître dans H. Bru, G. LABARre (éd.), L'Anatolie des peuples, des cités et des cultures (II millénaire av. J.-C.- $V^{e}$ siècle ap. J.-C.). Colloque international de Besançon - 26-27 novembre 2010, I, Besançon, p. 111-132.

Bekker-Nielsen T., 2008, Urban Life and Local Politics in Roman Bithynia. The Small World of Dio Chrysostomos, Aarhus (Black Sea Studies 7).

Bosch C., 1935, Die kleinasiatischen Münzen der römischen Kaiserzeit, II.1, Stuttgart.

Bosworth A. B., 1972, "Arrian's Literary Development», CQ, NS, 22, p. 163-185.

Bosworth A. B., 1980, A Historical Commentary on Arrian's History of Alexander, I, Oxford.

Bosworth A. B., 1988, From Arrian to Alexander. Studies in Historical Interpretation, Oxford.

Bosworth A. B., 1993, "Arrian and Rome: The Minor Works», ANRW, II.34.1, p. 226-275.

Boudon-Millot V., 2005, s.v. Ménodote de Nicomédie (M 133), DPhA, IV, p. 476-482.

Boulhol P., 1994, «L'apport de la hagiographie à la connaissance de la Nicomédie paléochrétienne (toponymie et monuments)», MEFRA, 10, p. 921-992.

Bounegru O., 2006, "Trafiquants et armateurs de Nicomédie dans la Méditerranée à l'époque romaine», dans L'Africa romana. Mobilità delle persone e dei popoli, dinamiche migratorie, emigrazioni ed immigrazioni nelle province occidentali dell'Impero romano. Atti del XVI convegno di studio, Rabat, 15-19 dicembre 2004, Rome, III, p. 1557-1568.

Bowersock G. W., 1969, Greek Sophists in the Roman Empire, Oxford.

Bowie E. L., 1970, «Greeks and their Past in the Second Sophistic», P\&P, 46, p. 3-41.

Burrell B., 2004, Neokoroi. Greek Cities and Roman Emperors, Leyde-Boston.

Conrad S., 2004, Die Grabstelen aus Moesia Inferior. Untersuchungen zu Chronologie, Typologie und Ikonographie, Leipzig.

Corsten Th., 2006, «Prosopographische und onomastische Notizen II », EA, 39, p. 121-132.

Corsten Th., 2010, "Names en -ıavó ç in Asia Minor. A Preliminary Study», dans R. W. V. CATLING, F. MARCHAND (éd.), Onomatologos. Studies in the Personal Names Presented to Elaine Matthews, Oxford, p. 456-463.

Dagron G., 1984, Constantinople imaginaire. Études sur le recueil des Patria, Paris.

DANA D., 2006, «Les noms de facture thrace dans LGPN IV : les noms fantômes et d'autres corrections », ZPE, 157, p. 127-142.

Dana M., 2011, Culture et mobilité dans le Pont-Euxin. Approche régionale de la vie culturelle des cités grecques, Bordeaux (Scripta Antiqua 37).

Dana M., 2012, «Pontiques et étrangers dans les cités de la mer Noire: le rôle des citoyennetés multiples dans l'essor d'une culture régionale», dans A. Heller, A.-V. Pont (éd.), Patrie d'origine et patries électives: les citoyennetés multiples dans le monde grec d'époque romaine, Bordeaux (Scripta Antiqua 40), p. 249-266.

Delivorrias A., 2000, A Guide to the Benaki Museum, Athènes.

Desideri P., 1994, s.v. Dion Cocceianus de Pruse dit Chrysostome (D 166), DPhA, II, p. 841-856.

Devine A. M., 1993, "Arrian's “Tactica” ", ANRW, II.34.1, p. 312-315. 
Dorandi T., 1994, s.v. Diogène de Nicomédie (D 142), DPhA, II, p. 806.

Dorandi T., 2000a, s.v. Euclide de Nicomédie (E 83), DPhA, III, p. 277.

Dorandi T., 2000b, s.v. Hérodote de Nicomédie (H 103), DPhA, III, p. 676.

Dörner Fr. K., 1952, Bericht über eine Reise in Bithynien. Ausgeführt im Jahre 1948 im Auftrage der Österreichischen Akademie der Wissenschaften, Vienne.

FERnoux H.-L., 2004, Notables et élites des cités de Bithynie aux époques hellénistique et romaine (III ${ }^{e}$ siècle av. J.-C.-III siècle ap. J.-C.). Essai d'histoire sociale, Lyon (CMO 31).

Follet S., 1976, Athènes au II et au III ${ }^{e}$ siècle. Études chronologiques et prosopographiques, Paris.

Follet S., 1989, s.v. Arrien de Nicomédie (A 425), DPhA, I, p. 597-604.

Follet S., 2000, s.v. Gellius de Corinthe (G 11), DPhA, III, p. 470-471.

Follet S., 2012, s.v. Quirinus (Q4), DPhA, Vb, p. 1793-1794.

Fuentes González P. P., 2000, s.v. Épictète (E 33), DPhA, III, p. 106-151.

Fuentes González P. P., 2012, s.v. Pontianus (P260), DPhA, Vb, p. 1286.

Goulet R., 1994, s.v. Démocrite de Nicomédie (D 70a), DPhA, II, p. 716.

Goulet R., 2005, s.v. Massalénos (Messalinus) (M 47), DPhA, IV, p. 302-303.

Grassl H., 1982, "Arrian im Donauraum», Chiron, 12, p. 245-252.

Gray V. J., 1990, «The Moral Interpretation of the "Second Preface” in Arrian's Anabasis», JRS, 110, p. 180-186.

Grélois J.-P., 1998, Dr. John Covel. Voyages en Turquie 1675-1677, Paris (Réalités byzantines 6).

Guerber É., 2009, Les cités grecques dans l'Empire romain. Les privilèges et les titres des cités de l'Orient hellénophone d'Octave Auguste à Dioclétien, Rennes.

Hannestad L., 1996, «This Contributes in No Small Way to One's Reputation: The Bithynian Kings and Greek Culture», dans P. BILDE et alii, Aspects of Hellenistic Kingship, Aarhus, p. 67-98.

Heller A., 2006, «Les bêtises des Grecs». Conflits et rivalités entre cités d'Asie et de Bithynie à l'époque romaine (129 a.C.-235 p.C.), Bordeaux.

Jones C.P., 2014, « Epigraphica X-XII », ZPE, 188, p. 28-34.

Kaloyeropoulou A. G., 1974, «Épitaphe mégarien[ne]», $A A A, 7$, p. 287-291.

LARonde A., 2002-2003, «Épigramme métrique de Lambèse», AntAfr, 38-39, p. 307-309.

MAdsen J. M., 2006, «Intellectual Resistance to Roman Hegemony and its Representativity», dans T. BEKKERNielsen (éd.), Rome and the Black Sea Region. Domination, Romanisation, Resistance, Aarhus (Black Sea Studies 5), p. 63-83.

Madsen J. M., 2009, Eager to be Roman. Greek Responses to Roman Rule in Pontus and Bithynia, Londres.

MAREK Chr., 2013, «Intellektuelle in Bithynien und ihre Feindschaften», dans E. Winter, K. ZimmermanN (éd.), Neue Funde und Forschungen in Bithynien. Friedrich Karl Dörner zum 100. Geburtstag gewidmet, Bonn (Asia Minor Studien 69), p. 33-46.

Marincola J.M., 1989, "Some Suggestions on the Proem and "Second Preface” of Arrian's Anabasis», JRS, 109, p. 186-189.

Merkelbach R., 1985, "Nikaia die Rankenreiche (E $\Lambda \mathrm{IK} \Omega \mathrm{PH})$. Ein übersehenes Fragment aus Arrians Bithyniaka»,EA, 5, p. 1-3 (= Philologica. Ausgewählte kleine Schriften, Stuttgart-Leipzig, 1997, p. 143-145).

Meritt B. D., 1963, « Greek Inscriptions», Hesperia, 32, p. 1-56.

Meyer E., 1897, s.v. Bithynia, RE, III.1, col. 507-524.

Moles J.L., 1985, «The Interpretation of the "Second Preface" in Arrian's Anabasis», JRS, 105, p. 162-168.

Oliver J.H., 1970, "Arrian and the Gellii of Corinth», GRBS, 11, p. 335-338.

Öztürk H. S., KIlıç Aslan S., 2012, «Nikaia'dan Yeni Yazıtlar II», Gephyra, 9, p. 101-110.

Puech B., 1994, s.v. Démétrios de Nicomédie (D 53), DPhA, II, p. 628.

Puech B., 2002, Orateurs et sophistes grecs dans les inscriptions d'époque impériale, Paris.

Puech B., 2005, s.v. Matinianus de Nicomédie (M 48), DPhA, IV, p. 303.

RADICKe J., 1999, FGrHistCont, IVA: Biography. 7 Imperial and Undated Authors, Leyde-Boston-Cologne. 
RÉmy B., 1989, Les carrières sénatoriales dans les provinces romaines d'Anatolie au Haut-Empire (31 av. J.-C.284 ap. J.-C.) (Pont-Bithynie, Galatie, Cappadoce, Lycie-Pamphylie et Cilicie), Istanbul-Paris (Varia Anatolica 2).

Roвert L., 1939, «Hellenica», RPh, p. 97-217 (= OMS, II, p. 1250-1370).

Robert L., 1960, Hellenica, 11-12, Paris.

Robert L., 1965, Hellenica, 13, Paris.

Robert L., 1966, Documents de l'Asie Mineure méridionale, Genève-Paris.

Robert L., 1977, «La titulature de Nicée et de Nicomédie. La gloire et la haine», HSPh, 81, p. 1-39 (=OMS, VI, p. 211-249; repris dans Choix d'écrits, Paris, 2007, p. 673-703).

Robert L., 1980, À travers l'Asie Mineure. Poètes et prosateurs, monnaies grecques, voyageurs et géographie, Athènes.

Robert L., 1987, Documents d'Asie Mineure, Athènes.

Rodríguez Horrillo M. Á., 2011, «El proemio a la Anábasis de Arriano», AION (filol.), 33, p. 77-88.

Ruge W., 1936, s.v. Nikomedeia, RE, XVII.1, col. 468-492.

SAmama É., 2003, Les médecins dans le monde grec. Sources épigraphiques sur la naissance d'un corps médical, Genève.

Schenkl H., 1989, Epicteti Dissertationes ab Arriano digestae. Accedunt fragmenta, Enchiridion ex recensione Schweighaeuseri, Gnomologiorum Epicteteorum reliquiae, Leipzig.

Schwartz E., 1895, s.v. Arrianus (9), RE, II.1, col. 1230-1247.

Silberman A., 1993, «Arrien, «Périple du Pont Euxin»: Essai d'interprétation et d'évaluation des données historiques et géographiques », ANRW, II.34.1, p. 276-311.

Solin H., 2002, "Analecta epigraphica CIC-CCVI», Arctos, 36, p. 107-142.

Stadter Ph. A., 1967, «Flavius Arrianus, the New Xenophon», GRBS, 8, p. 155-161.

Stadter Ph. A., 1980, Arrian of Nicomedia, Chapel Hill.

SwaIn S., 1996, Hellenism and Empire. Language, Classicism, and Power in the Greek World AD 50-250, Oxford.

Syme R., 1982, "The Carrer of Arrian», HSPh, 86, p. 181-211 (=Roman Papers, IV, Oxford, 1988, p. 21-49).

Syme R., 1985, «Correspondents of Pliny», Historia, 34, p. 324-359.

ŞAHIN S., 1974, Neufunde von antiken Inschriften in Nikomedeia (Izmit) und in der Umgebung der Stadt, Diss., Munich.

Tonnet H., 1988, Recherches sur Arrien, sa personnalité et ses écrits atticistes, I-II, Amsterdam.

Vidal-Naquet P., 1984, "Flavius Arrien entre deux mondes», dans Arrien. Histoire d'Alexandre. L'Anabase d'Alexandre le Grand et L'Inde, trad. P. SAvineL, Paris, p. 309-394.

Vitale M., 2012, Eparchia und Koinon in Kleinasien von der ausgehenden Republik bis ins 3. Jh. n. Chr., Bonn (Asia Minor Studien 67).

Vlassopoulos K., 2011, «Regional Perspectives and the Study of Greek History», IncidAntico, 9, p. 9-31.

Vlassopoulos K., 2013, Greeks and Barbarians, Cambridge.

Waddington W.H., continué par E. Babelon, Th. Reinach, 1908, Recueil général des monnaies grecques d'Asie Mineure, I.3, Nicée et Nicomédie, Paris.

Wirth G., 1964, «Anmerkungen zur Arrianbiographie: Appian-Arrian-Lukian», Historia, 13, p. $209-245$.

Wirth G., 1974, «Arrian und Traian - Versuch einer Gegenwartsdeutung», StudClas, 16, p. 169-209.

Withmarsh T. (éd.), 2010, Local Knowledge and Microidentities in the Imperial Greek World, Cambridge. 
